\title{
UNIVERSIDAD RUPERTO CAROLA DE HEIDELBERG: DESDE LA TRADICIÓN AL FUTURO
}

\author{
Walter Eckel*
}




\section{RESUMEN}

En una primera parte del artículo, como antecedente y contexto de las tradiciones y el prestigio de la Universidad de Heidelberg, pasaremos revista a algunos de los acontecimientos más importantes de su historia y de su proyección romántica universal, tan vinculadas a la ciudad que la alberga. En la segunda parte, analizaremos las razones por las que la Universidad de Heidelberg se cuenta entre las más importantes instituciones de excelencia académica de Alemania y de Europa, en materia de docencia e investigación, señalando además los conceptos y las estrategias innovadoras que se están aplicando para consolidar y proyectar su posición de líder mundial, como la creación del Heidelberg Center para América Latina, que se inaugurara en abril del presente año en Santiago de Chile.

\section{ABSTRACT}

In the first part of the paper, as part of the background data and the setting created by the tradition and the prestige of the University of Heidelberg, we shall review some of the most important events in its history and in its universal romantic projection, which are so closely linked to the city to which it belongs. In the second section, we shall analyze the reasons why the University of Heidelberg is among the most important institutions of academic excellence in Germany and in Europe with regard to teaching and research. We shall further describe the innovative strategies being applied to consolidate and project its position as a world leader, such as the creation of the Heidelberg Center for Latin America inaugurated in April 2002 in Santiago, Chile. 


\section{UNIVERSIDAD RUPERTO CAROLA DE HEIDELBERG: DESDE LA TRADICIÓN AL FUTURO}

\section{UNA MIRADA A LA HISTORIA}

Según las estadísticas, en el semestre de invierno 2002 / 2003 la Universidad de Heidelberg cuenta con más de 25.000 estudiantes. De ellos, 5.100, es decir el 20.3\%, son estudiantes extranjeros de 133 países. El grupo más numeroso proviene de otros países europeos; luego le siguen estudiantes de Asia y África. Más de 500 alumnos extranjeros realizan allí sus estudios de doctorado, cifra que representa casi un tercio de todos los estudiantes de doctorado matriculados en la Universidad de Heidelberg. ¿Qué motiva a tantos jóvenes de todas partes del mundo a estudiar y especializarse en Heidelberg?

El deseo de estudiar en una de las universidades más antiguas y de mayor prestigio del mundo, así como el de incorporarse a una tradición de docencia e investigación de más de seis siglos, constituyen motivaciones suficientes para quienes se proponen ingresar a la Universidad de Heidelberg. Sin embargo, lo que la mayoría de los estudiantes probablemente desconocen es que, desde el mismo año de sus fundación, la Universidad de Heidelberg comenzó a ejercer su gran poder de atracción más allá de las fronteras históricas.

Marsilius von Inghen, ex Rector de la Sorbona, quien a consecuencia del Cisma se vio obligado a abandonar la Universidad de París junto con profesores y estudiantes alemanes, fue el hombre escogido por Ruperto I para crear la Universidad de Heidelberg. En el año 1386, siguiendo el modelo de la Sorbona y bajo la rectoría de von Inghen, se iniciaron las actividades de docencia en las cuatro facultades tradicionales: Teología, Derecho, Medicina y Artes. Desde sus primeros años, la Universidad acuñó un gran prestigio entre los eruditos europeos, que terminó de consolidarse en el transcurso del siglo XV, sobre todo entre los estudiantes de Teología, que llegaban desde los más diversos países. A principios del siglo XVI, Heidelberg se había 
convertido en un importante centro del humanismo temprano en Alemania. En 1518, el fuerte impacto que tuvo la disputa de Martin Lutero en la Facultad de Teología indujo a muchos teólogos de Heidelberg a convertirse en impulsores de la Reforma en el suroeste de Alemania. El Catecismo de Heidelberg, redactado por profesores de dicha Facultad, sigue siendo hasta hoy la obra estándar fundamental de la Iglesia luterana. Por su parte, también la valiosa colección de 8.000 volúmenes reunida por la Universidad, conocida como "Biblioteca Palatina", acusó los efectos del Cisma: la Iglesia romana trasladó todo el acervo al Vaticano, en 1623.

Las pugnas no fueron inocuas: a fines del siglo XVII, Heidelberg y su Universidad habían perdido importancia y sólo hacia fines del siguiente siglo lograron recuperarse con la refundación de la ciudad, tras la Guerra de Sucesión del Palatinado. Surge con este "renacimiento" la imagen de la "Heidelberg romántica", que hoy siguen buscando académicos, estudiantes y turistas de todo el mundo. No sólo Goethe y Hölderlin fueron cautivados por la ciudad junto al río Neckar y por la imponente ruina de su castillo. Son muchos los testimonios de esta fascinación romántica: la recopilación del cancionero popular de Brentano y von Armin; el diario de von Eichendorff; obra de pintores como Fohr, Rottmannn, William Turner y de Graimsberg, narraciones de Mark Twain e incluso comedias musicales han contribuido a consolidar universalmente la fama de Heidelberg, de su Universidad y de sus arraigadas costumbres estudiantiles.

En el siglo XIX, reformada por el príncipe elector Karl Friedrich von Baden, la Universidad logró retomar definitivamente y luego proyectar hasta hoy la excelencia académica con que Heidelberg se ha consagrado en la historia universitaria mundial. Recuérdense en ciencias naturales los trabajos de Helmholtz que conducirían a la óptica fisiológica y a la acústica; el descubrimiento del análisis espectral por Kirchhoff y Bunsen; en medicina, los logros del Instituto de Investigación Experimental del Cáncer, que situaron a Heidelberg como líder en materia de investigación de este mal; en filosofía, la publicación de la "Enciclopedia básica de las Ciencias Filosóficas" de Hegel, devino en obra fundamental del pensamiento moderno; la edición de "Historia Alemana" de Treitschke, la fundación del periódico Deutsche Zeitung 
y la convocatoria de Thibaut a la creación del Código Civil alemán son sólo algunos de los hitos demostrativos de esta excelencia .

Del gran número de destacados científicos y académicos de las primeras décadas del siglo XX, abreviaremos mencionando sólo a Max Weber, quien influyó en forma decisiva en las ciencias sociales modernas. A pesar de ser un bastión de la burguesía protestante liberal, la Universidad de Heidelberg no logró sustraerse al influjo del nacionalsocialismo. Después de 1933, fueron expulsados de la docencia universitaria 59 académicos y empujados al exilio. En 1945, terminada la segunda guerra mundial, bajo la rectoría del médico Karl Heinrich Bauer, se logró retomar gradualmente las actividades lectivas. Con la creciente democratización del país, en los años cincuenta, volvieron también los primeros académicos y estudiantes extranjeros a Heidelberg. En los años siguientes, su número aumentó en la medida en que aumentó la integración política de la República Federal de Alemania en el contexto europeo y extraeuropeo. Como consecuencia de las reformas educacionales de los años sesenta el número de estudiantes registró un explosivo aumento. Como los edificios de la Universidad en el casco antiguo de la ciudad ya no daban abasto, se construyó el nuevo campus "Neuenheimer Feld" al otro lado del Neckar, en el cual se ubicaron preferentemente los institutos de Medicina y Ciencias Naturales. En esos años, se sentaron las bases para la orientación internacional e interdisciplinaria definitiva de la Universidad, que hoy la caracterizan más que nunca.

\section{EXCELENCIA ACADÉMICA}

Basta con recordar un par de datos para demarcar la excelencia académica lograda por Heidelberg: ocho de sus profesores han sido galardonados con premios Nobel; numerosos de sus científicos han recibido premios nacionales de investigación y otros tantos son miembros de honor de renombradas academias científicas alemanas y extranjeras. En lo relativo a la asignación de recursos de la Sociedad Alemana de Investigación Científica (Deutsche Forschungsgemeinschaft, DFG), Heidelberg ocupa un lugar privilegiado, a pesar de no ofrecer carreras de ingeniería. Asimismo, Heidelberg ocupa los primeros lugares en términos de la frecuencia con que sus académicos 
y científicos son citados en la literatura especializada en muchas disciplinas, y no es raro encontrar diferentes institutos de la Universidad de Heidelberg encabezando rankings internacionales de excelencia cada vez más importantes y numerosos. Finalmente, en cuanto a la entrega de becas a destacados profesores visitantes extranjeros de la Fundación Humboldt, la Universidad de Heidelberg ocupa el cuarto lugar dentro de Alemania.

Entre las razones que explican la posición de Heidelberg en el concierto universitario mundial, cabe destacar que cuenta con una de las mayores redes académicas de investigación en Alemania y en Europa. Esta oferta está compuesta como sigue:

Facultades de la Universidad de Heidelberg

- Teología,

- Derecho,

- Medicina

- Medicina Clínica (Mannheim)

- Filosofía Moderna

- Ciencias Económicas y Sociales

- Ciencias del Comportamiento y la Cultura

- Matemáticas e Informática

- Química y Geociencias

- Física y Astronomía

- Ciencias Biológicas

Junto con la amplia gama de programas de estudios en Humanidades, Ciencias Sociales, Ciencias Económicas, Ciencias Jurídicas, Ciencias Naturales y Medicina que culminan con los títulos licenciatura, diploma o Magister Artium, la Universidad creó, como parte de su estrategia de internacionalización, un número límitado de programas de Bachelor y Master, principalmente en el campo de las ciencias naturales.

Programas de master en inglés se ofrecen en las siguientes carreras internacionales:

- Application Oriented Computer Science

- Community Health and Health Management in Developing Countries

- Computer Linguistics 
- Molecular and Cellular Biology

- Molecular Biotechnology

- Physics

A lo anterior se agregan dos programas de master para estudiantes extranjeros dictados en alemán:

- Filología Alemana como lengua extranjera

- Derecho/Legum Magister (LLM)

Centros de Investigación

- Centro de Biología Molecular

- Centro de Bioquímica

- Centro Interdisciplinario de Ciencias Neurológicas

- Centro Interdisciplinario de Ciencias Informáticas

- Instituto del Sur Asiático

- 10 Centros Especiales de Investigación Conjunta

- 16 Colegios de Graduados para Doctorandos

Centros de Investigación Extrauniversitarios en Heidelberg

- Instituto Max-Planck de Derecho Extranjero Público y Derecho Internacional

- Instituto Max-Planck de Investigación Médica

- Instituto Max-Planck de Física Nuclear

- Instituto Max-Planck de Astronomía

- Centro Alemán de Investigación del Cáncer

- Laboratorio Europeo de Biología Molecular

El gran número de estos "centros de excelencia", tan cerca el uno del otro en una ciudad de apenas 140.000 habitantes, crea una densa red de cooperaciones académico-científicas y fomenta el trabajo interdisciplinario. El hecho de que una parte de los científicos de los Centros de Investigación Extrauniversitarios trabajen simultáneamente como profesores en las diferentes facultades de la Universidad, permite entregar un apoyo óptimo a los jóvenes científicos. Éste es también el objetivo de los 16 Colegios de Graduados para Doctorandos apoyados por la Sociedad Alemana de Investigación (DFG), con los cuales la Universidad de Heidelberg ocupa el primer lugar en toda la República Federal. Asimismo, el intercambio de estudiantes y graduados con 
más de 250 universidades en todo el mundo, ayuda a establecer una amplia red de estrecha cooperación científica.

La Universidad de Heidelberg se entiende como una "Universidad con amplia gama de carreras orientadas a la investigación", en que se encuentran representados todos los ramos de las ciencias naturales y las humanidades; se incluyen especialmente las así llamadas "disciplinas menores", como Ciencias Orientales y Ciencias Antiguas, las que, aunque desde la perspectiva de su dotación de personal y su equipamiento -y a veces también por su número de estudiantes- son pequeñas, tienen empero una importancia que no debe subestimarse, en términos sociales y de contenido: conservan la memoria cultural y, a través del análisis crítico del pasado de nuestra sociedad, entregan orientaciones para el futuro y contribuyen a la pluralidad del pensamiento. En estas carreras, al igual que en las otras más grandes en cuanto a número de estudiantes, rige el principio de la unidad entre docencia e investigación. Los resultados de la investigación son integrados en los contenidos y en los métodos de la docencia; a los estudiantes se les enseña tempranamente a realizar sus propias investigaciones. El objetivo es formar a la nueva generación de académicos en el contacto con científicos de otras disciplinas. Esta interacción no sólo pretende contribuir a una mejor comprensión de la propia disciplina y de disciplinas relacionadas; también se propone ayudar a concebir estrategias de solución para los problemas. A partir de una sólida investigación básica se desarrollan las capacidades para la investigación aplicada y la transferencia de conocimientos.

Con la finalidad de representar los intereses de las universidades orientadas a la investigación frente a la Unión Europea y a los gobiernos de los países integrantes, pero también para asesorarlos en materias de investigación, la Universidad de Heidelberg se ha asociado con las siguientes universidades para formar la League of European Research Universities (LERU): Cambridge, Edimburgo, Ginebra, Helsinki, Karolinska (Estocolmo, Suecia), Leiden, Leuven, Munich, Milán, Oxford y Estrasburgo.

Si bien el énfasis está en la investigación básica, Heidelberg -como centro importante de actividad científica, provisto de gran cantidad de institutos de investigación y numerosas empresas químicas, 
farmacéuticas e informáticas con fuerte capacidad financiera- ofrece excelentes condiciones para formas innovadoras de cooperación. Los centros científicos universitarios y extrauniversitarios realizan actividades de investigación básica en áreas claves del siglo XXI: ciencias neurológicas, biología molecular, investigación del cáncer, medicina, física, química e informática con orientación científica. Los contactos del mundo académico-científico con las empresas de la región Rhein-Neckar producen considerables efectos sinérgicos. Para la ciencia ello significa nuevos impulsos de investigación; a las empresas, por su parte, les brinda acceso a los últimos conocimientos científicos que son de gran utilidad para el desarrollo de productos. Este potencial es aprovechado por el Parque Tecnológico, en rápida expansión y ubicado junto al nuevo campus universitario, en el cual se han establecido numerosas empresas emergentes. A partir de la estrecha cooperación con el sector privado se están generando puestos de trabajo atractivos para los jóvenes científicos. En tiempos de escasez de empleo, éste es ciertamente un efecto secundario bienvenido, consecuencia de la interrelación entre investigación básica e investigación aplicada.

Hemos visto cómo la ciudad y la Universidad de Heidelberg son capaces de convocar a gran cantidad de estudiantes, académicos y científicos alemanes y extranjeros. Por una parte, este hecho imprime un ambiente cosmopolita a la ciudad, pero, por otra parte, implica enormes desafíos de organización. Se requiere disponer de alojamientos y asesoría, así como de cursos de alemán para 5.000 estudiantes, académicos y científicos internacionales. Ésta es la misión del Servicio Estudiantil (Studentenwerk), de la Oficina de Asuntos Internacionales (Akademisches Auslandsamt) y del Centro de Estudios Internacionales, que para estos efectos se coordina con las diferentes facultades e institutos. La experiencia de estudiar, investigar y dar clases en Heidelberg marcará para toda la vida, como se comprueba en los devenires profesionales de quienes allí han estado y en instituciones como "Heidelberg Alumni International", constituida por ex-alumnos. El que la Universidad no se ha quedado descansando en estos laureles sino que, por el contrario, está buscando nuevas estrategias complementarias de internacionalización que le permitan mantenerse vigente en la competencia internacional entre las universidades, lo demuestran algunas de sus iniciativas más recientes. Con la fundación, en Santiago de Chile, del Heidelberg Center para América Latina, se 
inició una experiencia completamente nueva: es la primera Universidad alemana que crea un centro de estudios de postgrado y de postítulo fuera de las fronteras nacionales. Este centro fue inaugurado oficialmente el 9 de abril de 2002 por el Rector de la Universidad de Heidelberg, Prof. Dr. Peter Hommelhoff y el Embajador de la República Federal de Alemania, señor Georg Clemens Dick, con asistencia de las máximas institutiones y autoridades del mundo de la educación y la cultura en Chile, así como destacadas personalidades, empresariales, políticas y académicas de ambos países.

La misión de este centro de estudios, operado en cooperación con la Pontificia Universidad Católica de Chile y la Universidad de Chile, es la exportación de la oferta académica de Heidelberg a Chile y Latinoamérica. En este primer año de funcionamiento, junto con un programa de Master on European Political Studies que se ofrece en dos versiones, de un año y dos años respectivamente, se han realizado además seminarios de especialización en áreas de medicina, psicología y ciencias sociales.

El Heidelberg Center para América Latina está concebido como una institución al servicio del diálogo académico-científico y será un modelo para proyectos similares en otras partes del mundo. La exportación de la oferta académica de Heidelberg, como importante elemento de la estrategia de modernización e internacionalización de esta Universidad, está orientada a fortalecer su posición en el contexto mundial de la competencia entre universidades y a compartir tradiciones de excelencia con países interlocutores que así lo entiendan. Sin duda, esta experiencia innovadora constituye un gran esfuerzo y un desafío sólo asumible mediante la fuerza que le confieren sus más de 600 años de historia y la voluntad de innovación de sus miembros, consecuentemente con al lema escogido para la conmemoración de los 600 años de su fundación: "Desde la tradición al futuro". 